\title{
Model Calculations of Elastic Moduli in Highly Deformed Composites
}

\author{
H. J. BUNGE \\ Department of Physical Metallurgy, TU Clausthal, FRG
}

(Received 24 June 1988)

Young's modulus of heavily deformed two-phase composites shows an unusually high increase after plastic deformation. It is assumed that this is due to two reasons, i.e. texture changes and changes of the moduli of the constitutive phases on the basis of non-linear elasticity theory and internal stresses of opposite sign in the phases. Expressions of the two contributions are given on the basis of simple model assumptions. It is estimated that the changes of shape and arrangement of the phases and shape and arrangement of the crystallites in the phases are only of minor importance.

KEY WORDS: Composite materials, Anomalous Young's modulus, Residual stresses, Non-linear elasticity, Texture mean values.

\section{INTRODUCTION}

Composite materials can be produced by powder metallurgical methods. Thereby fine powders of the component materials are mixed, compacted and extruded at an appropriate temperature with deformation degrees in the range of 70-90\% (see e.g. Wassermann 1981). This latter step is essential since it is the reason for the formation of a compact composite. The so obtained materials can be further deformed by rolling or wire drawing. Materials of this type consist of two (or more) phases the particles of which are elongated in one direction (fibres) or in two directions (bands). The 
particles themselves are polycrystalline with, usually, strong textures.

Composites of this type exhibit a number of "anomalous" properties which means that these properties cannot be understood on the basis of the properties of the components, alone. Rather, the anomalies are due to phase interactions of various kinds. One of these anomalous properties is an unexpectedly strong increase of Young's modulus with the degree of deformation (Welch, Ratke, Wassermann 1984).

The elastic properties of a multiphase, polycrystalline material may, in principle, depend on the following structural parameters:

1. The macroscopic properties are the weighted mean values of the properties of the phases.

1.1 These mean values depend on the shape and arrangement of the phase particles.

2. The elastic properties of the particles themselves will, in general, be anisotropic. They are the orientation mean values of the crystallographic anisotropies of the corresponding crystallites.

2.1 These mean values, too, depend on the shape and arrangement of the crystallites within the particles (phases).

3. Finally, the elastic moduli of the constitutive crystallites themselves may deviate from their ideal values measured in big single crystals. This latter effect may be due, for instance, to:

3.1 lattice defects, e.g. dislocation substructures

3.2 internal stresses

This latter influence will be particularly discussed in the following.

The structural variable of point 1 is the volume fractions $x^{\alpha}$ of the phases $(\alpha)$ by which the mean values are to be weighted. Usually the linear mean value is considered but also the higher order terms of $x^{\alpha}$ may be included. If we consider only the variation of the elastic moduli with the degree of deformation, this term remains constant.

The shape and arrangement of the particles can be described by particle shape functions and phase correlation functions which have, however, not yet uniquely been defined nor are they taken 
quantitatively into account in a rigorous theory. The influence of shape and arrangement of the phases is generally smaller then the influence of their volume fractions. It expresses itself in the higher order terms of $x^{\alpha}$ in the mean value formula. Furthermore, if only the variation of the elastic moduli is considered then only the variation of this higher order effect may enter. Hence, the contribution of this effect is also considered to be small.

The controlling variable in point 2 is the textures of the phases. The textures are changed by deformation. This holds also for high deformation degrees were the type of texture is generally well developed. Nevertheless, the degree of the texture-expressed for instance in the texture index-may still increase. Hence, the contribution of texture changes to the variation of the elastic moduli cannot be neglected.

It has also been found that the textures of the phases in multiphase composites may be strongly different from textures in single phase materials with the same degree of deformation (Wassermann, Bergmann, Frommeyer 1978). Furthermore, texture measurements by $x$-ray diffraction in multiphase materials may suffer from the effect of anisotropic absorption (Bunge, Liu, Hanneforth, 1987). Hence, quantitative texture measurements in deformed composites must be carried out by neutron diffraction (Brokmeier, Böcker, Bunge in print) which has not yet been done for the above mentioned $\mathrm{Fe}-\mathrm{Ag}$ composites.

The elastic moduli $\tilde{C}$ of a textured single phase polycrystalline material can be calculated in a good approximation using the Hill average of the Voigt and the Reuss mean values (Bunge 1968, 1974, Morris, 1969, 1974). For the general case of non-equiaxed grains, Hill's assumption must be formulated

$$
\tilde{C}=m \cdot \bar{C}^{v}+(1-m) \bar{C}^{R}
$$

whereby $m=0.5$ corresponds to the original Hill approximation which is assumed to be valid in equiaxed grain structures (Hill 1952). In highly deformed composites this assumption cannot be made. Nevertheless, the value of $m$ can be assumed to be near to 0.5 and its variation with the degree of deformation will also not be very strong. Furthermore, the difference between $\bar{C}^{v}$ and $\bar{C}^{R}$ itself is only in the order of 10-20\%. Hence, a change of $m$ may result in a variation of $\tilde{E}$ of a few percent only. 
The influence of lattice defects on the elastic properties of crystals is in principle well known. The quantitative dependence on the details of e.g. dislocation substructures is, however, not very well known. The development of dislocation substructures in highly deformed composites has been found different from corresponding single phase materials. Smaller dislocation cells were found in composites. On the other hand, very small particles in composites were found essentially dislocation free. Hence, it is difficult to estimate a contribution of changing substructure on the variation of the elastic moduli upon deformation.

Finally, the influence of the last one of the parameters, listed above, i.e. internal stresses, can be quantified more easily. When a composite is being deformed, the necessary stresses in the phases will be different according to the different yield strengths (including effects like dislocation hardeneing, texture hardeneing or softening, boundary influences expressed by a Hall-Petch relation and others). After deformation the applied stresses will relax when no more external stress is present. In this state the phases will develop internal stresses of opposite sign and rather high absolute values (see e.g. Cohen 1986). This requires to calculate the average elastic moduli of the stressed particles on the basis of non-linear elasticity. The same effect has been assumed by Janowski and Tsakalakos (1985) for layered two-component materials.

\section{NON-LINEAR MEAN VALUES OF THE ELASTIC MODULI}

In a simple model, we assume a two-phase material consisting of the phases $\alpha$ and $\beta$ with the volume fractions $x^{\alpha}$ and $x^{\beta}$

$$
x^{\alpha}+x^{\beta}=1
$$

The composite may be deformed plastically whereby a stress-strain relation $\bar{\sigma}(\bar{\varepsilon})$ holds. Similarly, the stress-strain relations of the two phases be $\sigma^{\alpha}\left(\varepsilon^{\alpha}\right)$ and $\sigma^{\beta}\left(\varepsilon^{\beta}\right)$. If the yield strengths of the phases are different, the phases will undergo different strains $\varepsilon^{\alpha}$ and $\varepsilon^{\beta}$ which are somehow related to the average strain $\bar{\varepsilon}$

$$
\varepsilon^{\alpha}=\varepsilon^{\alpha}(\bar{\varepsilon}) ; \quad \varepsilon^{\beta}=\varepsilon^{\beta}(\bar{\varepsilon})
$$

The latter one may be assumed to be the linear mean value of the 
strains and phases

$$
\bar{\varepsilon}=x^{\alpha} \cdot \varepsilon^{\alpha}+x^{\beta} \cdot \varepsilon^{\beta}
$$

These strains contain elastic and plastic parts which need not to be distinguished here. In the present considerations, the specific forms of the functions in Eq. (3) do not matter. They allow, however, to express $\sigma^{\alpha}$ and $\sigma^{\beta}$ as functions of $\bar{\varepsilon}$, i.e. $\sigma^{\alpha}(\bar{\varepsilon})$ and $\sigma^{\beta}(\bar{\varepsilon})$. The mean stress of the composite is assumed to be the linear mean value

$$
\bar{\sigma}(\bar{\varepsilon})=x^{\alpha} \cdot \sigma^{\alpha}(\bar{\varepsilon})+x^{\beta} \cdot \sigma^{\beta}(\bar{\varepsilon})
$$

After deformation the macroscopic stress $\bar{\sigma}$ is relaxed to zero. Then Eq. (5) refers to the residual stresses $\sigma_{r}^{\alpha}$ and $\sigma_{r}^{\beta}$ in the two phases. It is

$$
x^{\alpha} \cdot \sigma_{r}^{\alpha}+x^{\beta} \cdot \sigma_{r}^{\beta}=0
$$

We assume, for the sake of simplicity, that this relaxation process is completely elastic as is shown in Figure 1. Then each phase undergoes the same elastic redeformation $\Delta \varepsilon$ which is also equal to the macroscopic redeformation. Within the linear elasticity theory it is

$$
\begin{aligned}
& \sigma^{\alpha}-\sigma_{r}^{\alpha}=C^{\alpha} \cdot \Delta \varepsilon \\
& \sigma^{\beta}-\sigma_{r}^{\beta}=C^{\beta} \cdot \Delta \varepsilon
\end{aligned}
$$

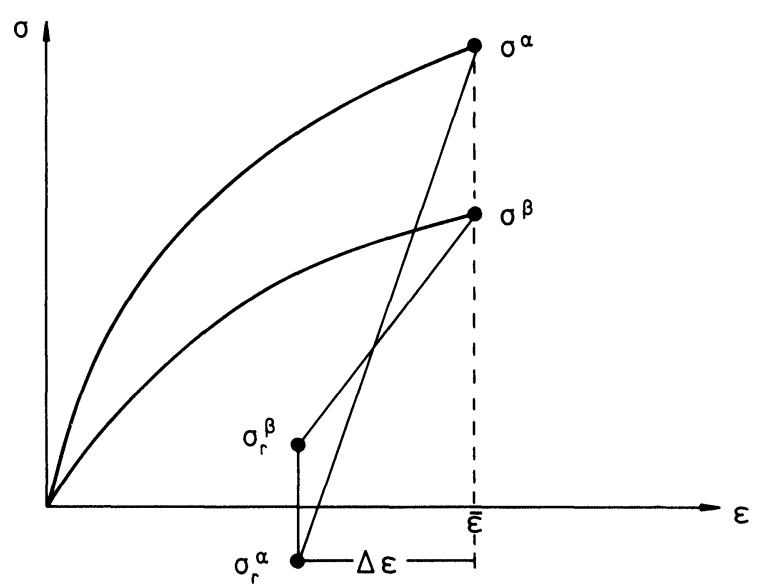

Figure 1 Plastic stress-strain curves of the phases of a two-phase composite (schematically) and the elastic relaxation. 
Where $C^{\alpha}$ and $C^{\beta}$ are the linear elastic stiffness tensors of the phases $\alpha$ and $\beta$. With Eq. (5) and (6) one obtains

$$
\bar{\sigma}=\bar{C} \cdot \Delta \varepsilon
$$

with

$$
\bar{C}=x^{\alpha} \cdot C^{\alpha}+x^{\beta} \cdot C^{\beta}
$$

Eq. (8) may be inverted

$$
\Delta \varepsilon=\bar{S} \cdot \bar{\sigma}
$$

where $\bar{S}$ is the elastic compliance tensor corresponding to $\bar{C}$. This allows the residual stresses to be expressed in terms of the average stress

$$
\begin{aligned}
\sigma_{r}^{\alpha} & =\sigma^{\alpha}-C^{\alpha} \cdot \bar{S} \cdot \bar{\sigma} \\
\sigma_{r}^{\beta} & =\sigma^{\beta}-C^{\beta} \cdot \bar{S} \cdot \bar{\sigma}
\end{aligned}
$$

Since the stresses $\sigma^{\alpha}$ and $\sigma^{\beta}$ reached during deformation are not very well known, anyway, it seems justified to use linear elasticity theory in Eq. (7) although we shall use later on the non-linear theory for the smaller residual stresses.

The elastic properties of the composite in the relaxed state may be expressed in the Voigt approximation

$$
\bar{C}^{\prime}=x^{\alpha} \cdot C^{\prime \alpha}+x^{\beta} \cdot C^{\prime \beta}
$$

Now, we assume second order elasticity theory (see e.g. Haussühl, 1983, Hearmon, 1979) putting

$$
\begin{aligned}
& C^{\prime \alpha}=C^{\alpha}+\dot{C}^{\alpha} \cdot \sigma_{r}^{\alpha} \\
& C^{\prime \beta}=C^{\beta}+\dot{C}^{\beta} \cdot \sigma_{r}^{\beta}
\end{aligned}
$$

This is illustrated in Figure 2. The average according to Eq. (13) is given by

$$
\bar{C}^{\prime}=\bar{C}+x^{\alpha} \cdot \dot{C}^{\alpha} \cdot \sigma_{r}^{\alpha}+x^{\beta} \cdot \dot{C}^{\beta} \cdot \sigma_{r}^{\beta}
$$

and with Eq. (6)

$$
\bar{C}^{\prime}=\bar{C}+x^{\alpha} \cdot \sigma_{r}^{\alpha}\left[\dot{C}^{\alpha}-\dot{C}^{\beta}\right]
$$

or with Eq. 11

$$
\bar{C}^{\prime}=\bar{C}+x^{\alpha} \cdot\left[\sigma^{\alpha}-C^{\alpha} \cdot \bar{S} \cdot \bar{\sigma}\right] \cdot\left[\dot{C}^{\alpha}-\dot{C}^{\beta}\right]
$$




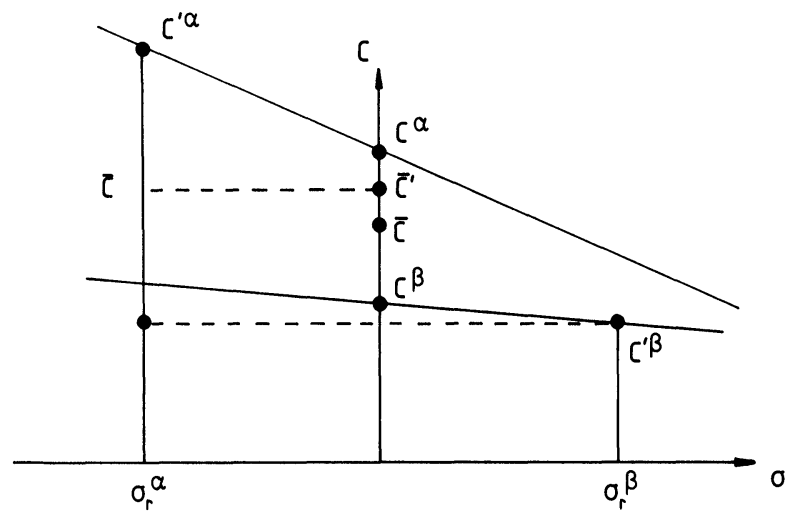

Figure 2 Mean value of Young's modulus in a two-phase composite assuming stress-dependent moduli in the two phases.

The stress derivatives $\dot{C}$ of the elastic constants used in Eq. (14) and (15) can be expressed by the third order elastic stiffnesses in the following way. With the definition

$$
\sigma=C \cdot \varepsilon+C^{(3)} \cdot[\varepsilon]^{2}
$$

one obtains

$$
C^{\prime}=\frac{\partial \sigma}{\partial \varepsilon}=C+2 \cdot C^{(3)} \cdot \varepsilon
$$

and replacing $\varepsilon$ by $\sigma$ in a linear approximation one obtains

$$
C^{\prime}=C+2 \cdot C^{(3)} \cdot S \cdot \sigma
$$

Hence, it follows

$$
\dot{C}=2 \cdot C^{(3)} \cdot S
$$

where $C^{(3)}$ are the third order stiffnesses and $S$ are the second order compliances.

It is seen $(E q .16,17)$ that in the case of internal stresses and non-linear elasticity theory the mean value of the elastic moduli contains a term which depends on the internal stresses and which may thus increase with deformation due to increasing stresses. The third order elastic constants are numerically not very well known. Hence, only the order of magnitude of the expected effect can be 
estimated. In the Landolt-Börnstein data collection compiled by Hearmon (1979), values of $C_{111}$ for iron are given which range from -4820 to $-2830 \mathrm{GPa}$, the reported value for silver is $-843 \mathrm{GPa}$. Using these values and the values $S_{11}$ for $S$ in Eq. (22) (i.e. $7.67(\mathrm{TPa})^{-1}$ and $22.9(\mathrm{TPa})^{-1}$ respectively) one obtains values of $\left(\dot{C}^{\mathrm{Fe}}-\dot{C}^{\mathrm{Ag}}\right)$ ranging from 4.8 to 53 , which seams quite reasonable. The increase of Young's modulus $\left(\bar{C}^{\prime}-\bar{C}\right) / \bar{C}$ found by Welch, Ratke and Wassermann (1984) was in the order of magnitude of 0.4 at $94 \%$ rolling reduction. According to $\mathrm{Eq}$. (17) this requires residual compressive stresses $\sigma_{r}^{\mathrm{Fe}}$ in the iron phase in the order of magnitude of $0.8 \%$ to $8 \%$ of the average Young's modulus $\bar{C}$. Internal stresses in the order of magnitude of $1 \%$ of Young's modulus were frequently found in single phase materials (see e.g. Macherauch and Hauk 1983). Internal stresses in two-phase materials must be assumed to be even higher (e.g. Cohen 1986). Hence, the estimated order of magnitude seems to be quite reasonable.

It is, thus, possible to understand an increase of $40 \%$ of Young's modulus upon rolling on the basis of non-linear elasticity. On the other hand, also texture changes must still be assumed in this range although quantitative texture measurements and calculations of Young's modulus on this basis were not yet carried out in the above-mentioned case. The texture effect and the internal stress effect may be additive or substractive in certain cases which may account for different results of the magnitude of increase of Young's modulus in two-phase compositers (e.g. Bevk et al. 1978, Bevk 1983).

\section{TEXTURE MEAN VALUES}

The quantities $C^{\alpha}, C^{\beta}$ and $\dot{C}^{\alpha}, \dot{C}^{\beta}$ are texture mean values of the corresponding single crystal quantities taken with the two textures $f^{\alpha}(g), f^{\beta}(g)$ of the two phases. If we assume that grain shape in the two phases does not change very much during deformation, i.e. $m=$ const in Eq. (1), then the texture mean values of $C$ and $\dot{C}$ in Eq. $(14,15)$ can be calculated with either of the two approximations according to Voigt or Reuss.

In order to calculate the orientation mean values, the tensors $C$ and $\dot{C}$ must be referred to a common sample coordinate system $K_{A}$. 
The crystal coordinate system $K_{B}$ of a considered crystal may be related to $K_{A}$ by the rotation $g$

$$
K_{B}=g \cdot K_{A} ; \quad g=\left[g_{i j}\right]
$$

The components of the tensors in the crystal coordinate system be $C_{i j k l}^{0}, \dot{C}_{i j k l m n}^{0}$. The components in the system $K_{A}$ are then given by the transformation relations

$$
\begin{gathered}
C_{a b c d}(g)=C_{i j k l}^{0} \cdot g_{a i} \cdot g_{b j} \cdot g_{c k} \cdot g_{d l}=C_{i j k l}^{0} \cdot T_{a b c d}^{i j k l} \\
\dot{C}_{a b c d e f}(g)=\dot{C}_{i j k l m n}^{0} \cdot g_{a i} \cdot g_{b j} \cdot g_{c k} \cdot g_{d l} \cdot g_{e m} \cdot g_{f n}=\dot{C}_{i j k l m n}^{0} \cdot T_{a b c d e f}^{\prime i j k l m n}
\end{gathered}
$$

where $T$ and $T^{\prime}$ are orientation factors and the Einstein summation is being used. The orientation mean values of these quantities are given by

$$
\begin{gathered}
\bar{C}_{a b c d}=C_{i j k l}^{0} \cdot \bar{T}_{a b c d}^{i j k l} \\
\bar{C}_{a b c d e f}=\dot{C}_{i j k l m n}^{0} \cdot \bar{T}_{a b c d e f}^{\prime i j k l m n}
\end{gathered}
$$

where $\bar{T}$ and $\bar{T}^{\prime}$ are texture factors defined by

$$
\begin{gathered}
\bar{T}_{a b c d}^{i j k l}=\oint T_{a b c d}^{i j k l}(g) \cdot f(g) \cdot d g \\
\bar{T}_{a b c d e f}^{\prime i j k l m n}=\oint T_{a b c d e f}^{i j k l m n}(g) \cdot f(g) \cdot d g
\end{gathered}
$$

The texture may be given in terms of the series expansion (see e.g. Bunge 1982)

$$
f(g)=\sum_{\lambda=0}^{\infty} \sum_{\mu=1}^{M(\lambda)} \sum_{v=1}^{N(\lambda)} C_{\lambda}^{\mu \nu} \cdot \dot{T}_{\lambda}^{\mu \nu}(g)
$$

Also the orientation factors $T$ and $T^{\prime}$ of Eq. $(24,25)$ can be expressed in terms of these functions

$$
\begin{gathered}
T_{a b c d}^{i j k l}(g)=\sum_{\lambda=0}^{4} \sum_{\mu=1}^{M(\lambda)} \sum_{v=1}^{N(\lambda)} t_{\lambda}^{\mu v}\left(\begin{array}{l}
i j k l \\
a b c d
\end{array}\right) \cdot \dot{T}_{\lambda}^{\mu v}(g) \\
T_{a b c d e f}^{\prime i j k l m n}(g)=\sum_{\lambda=0} \sum_{\mu=1}^{M(\lambda)} \sum_{v=1}^{N(\lambda)} t_{\lambda}^{\prime \mu v}\left(\begin{array}{l}
i j k l m n \\
a b c d e f
\end{array}\right) \cdot \dot{T}_{\lambda}^{\mu \nu}(g)
\end{gathered}
$$


Then the texture coefficients $\bar{T}$ and $\bar{T}^{\prime}$ are given by

$$
\begin{aligned}
\bar{T}_{a b c d}^{i j k l} & =\sum_{\lambda=0}^{4} \sum_{\mu=1}^{M(\lambda)} \sum_{v=1}^{N(\lambda)} \frac{1}{2 \lambda+1} \cdot t_{\lambda}^{\mu \nu}\left(\begin{array}{l}
i j k l) \\
\text { abcd }
\end{array}\right) \cdot C_{\lambda}^{\mu \nu} \\
\bar{T}_{a b c d e f}^{\prime i j k l m n} & =\sum_{\lambda=0}^{6} \sum_{\mu=1}^{M(\lambda)} \sum_{\nu=1}^{N(\lambda)} \frac{1}{2 \lambda+1} \cdot t_{\lambda}^{\prime \mu v}\left(\begin{array}{l}
i j k l m n \\
\text { abcdef })
\end{array}\right) \cdot C_{\lambda}^{\mu \nu}
\end{aligned}
$$

The purely mathematical quantities $t_{\lambda}^{\mu \nu}$ have been derived by Morris (1969) in a quite general way and in a more specific form for cubic orthorhombic symmetry by Bunge $(1968,1974)$. The quantities $t^{\prime}$ have not yet been given numerically.

In the case of cubic orthorhombic symmetry Eq. (33) contains only three texture coefficients, whereas Eq. (34) contains seven. Assuming not too high elastic anisotropy, and restricting ourselves to directions in the sheet plane $\left(\alpha=90^{\circ}\right)$, Young's modulus can be expressed in the simple form

$$
\bar{E}^{V, R, H}(\beta)=\bar{E}_{\text {random }}^{V, R, H}+E^{a} \cdot \varphi^{4}(\beta)
$$

where

$$
E^{a}=C_{11}^{0}-C_{12}^{0}-2 \cdot C_{44}^{0}
$$

is the elastic anisotropy and

$$
\varphi^{4}(\beta)=a^{1} \cdot C_{4}^{11}+a^{2} \cdot C_{4}^{12} \cdot \sin 2 \beta+a^{3} \cdot C_{4}^{13} \cdot \sin 4 \beta
$$

is an anisotropy function, the coefficients $a^{i}$ of which are purely mathematical constants. A similar expression can be obtained for the stress dependence $\dot{E}$ of Young's modulus (analogous to $\dot{C}$ in Eq. $14,15)$

$$
\dot{E}^{V, R, H}(\beta)=\dot{E}_{\text {random }}^{V, R, H}+\dot{E}^{a} \cdot \varphi^{4}(\beta)+\dot{E}^{b} \cdot \varphi^{6}(\beta)
$$

with

$$
\begin{gathered}
\dot{E}^{a}=\dot{C}_{111}^{0}-\dot{C}_{112}^{0}-4 \dot{C}_{155}^{0} \\
\dot{E}^{b}=\dot{C}_{111}^{0}-3 \cdot \dot{C}_{112}^{0}-12 \cdot \dot{C}_{155}^{0}+12 \cdot \dot{C}_{144}^{0}+2 \cdot \dot{C}_{123}^{0}+16 \cdot \dot{C}_{456}^{0}
\end{gathered}
$$

and

$$
\begin{aligned}
\varphi^{6}(\beta)= & b_{4}^{1} C_{4}^{11}+b_{4}^{2} C_{4}^{12} \sin 2 \beta+b_{4}^{3} C_{4}^{13} \sin 4 \beta+b_{6}^{1} C_{6}^{11} \\
& +b_{6}^{2} C_{6}^{12} \sin 2 \beta+b_{6}^{3} C_{6}^{13} \sin 4 \beta+b_{6}^{4} C_{6}^{14} \sin 6 \beta
\end{aligned}
$$

Also in Eq. (41) the $b_{i}^{j}$ are purely mathematical constants. 
In the case of cubic crystal symmetry and axial sample symmetry, the function $\varphi(\beta)$ is to be replaced by another function $\varphi^{\prime}(\alpha)$ where $\alpha$ is the angle to the axis direction

$$
\varphi^{\prime}(\alpha)=a^{\prime} \cdot C_{4}^{11} \cos 2 \alpha
$$

In this case, only one texture coefficient is involved.

\section{CONCLUSIONS}

The observed, rather high increase of Young's modulus of twophase composites after plastic deformation may have several reasons. Estimations show that the changes of the geometrical structural parameters such as the shape and arrangement of the particles of the phases and the shape and arrangement of the crystallites in the phases is only a second order effect, the contribution of which is assumed to be small.

Internal stresses in the phases after deformation will have opposite sign in the two phases. If it is assumed that they reach an order of magnitude of $1 \%$ of Young's modulus then the stress dependence of the elastic constants must be taken into account. A variation of Young's modulus in the order of $40 \%$ as was observed, may thus be, in principle, understood.

A second contribution to the variation of Young's modulus with deformation will certainly be due to texture changes. In this respect, the influence of anisotropic absorption of $x$-rays in anisotropic two-phase materials must be taken into account. This effect may strongly falsify texture measurements by $x$-rays. Quantitative texture measurements in such materials must thus be carried out by neutron diffraction where this effect does not occur.

The contribution of textures changes and that of residual stresses to the macroscopic Young's modulus may in principle be additive or substractive which may account for the different results found in different composites.

\section{References}

Bevk, J., Harbinson, J. P., Bell, J. L. (1978). J. Appl. Phys. 49, 6031-6038.

Bevk, J. (1983). Ann. Rev. Mater. Sci. 13, 319-338. 
Brokmeier, H. G., Böcker, W. and Bunge, H. J. (1988). Textures and Microstructures 8 and 9, 429-441.

Bunge, H. J. (1968). Kristall u. Technik 3, 431-438.

Bunge, H. J. (1974). Kristall u. Technik 9, 413-423.

Bunge, H. J. (1982). Texture Analysis in Materials Science, Butterworth Publ. London.

Bunge, H. J., Liu, Y. S. and Hanneforth, R. (1987). Scripta Met. 21, 1423-1427.

Cohen, J. B. (1986). Powder Diffraction 1 No 2, 15-21.

Haussühl, S. (1983). Kristallphysik, Physikverlag, Weinheim.

Hearmon, R. F. S. (1979). The third- and higher order elastic constants. In: Landolt Börnstein Vol. III/11, Springer Verlag, Berlin.

Hill, R. (1952). Proc. Phys. Soc. A65, 349-354.

Janowski, A. F. and Tsakalakos, T. (1985). Journ. Phys. F. Metal Physics 15, 1279-1292.

Morris, P. R. (1969). J. Appl. Phys. 40, 447-448.

Morris, P. R. (1970). Int. Eng. Sci. 8, 49-61.

Ratke, L. Textures and Microstructures (to be published).

Wassermann, G., Bergmann, H. W. and Frommeyer, G. (1978). Proceedings of the Fifth International Conference on Textures of Materials (ICOTOM 5). Ed. G. Gottstein and K. Lücke. Springer Verlag, Berlin p. 37-46.

Wasserman, G. (1981). In: Verbundwerkstoffe. DGM Informationsgesellschaft Oberursel 63-67.

Welch, P. I., Ratke, L. and Wassermann, G. (1984). Proceedings of the Seventh International Conference on Textures and Materials ICOTOM 7. Ed. C. M. Brakman, R. Jongenburger, E. J. Mittemeyer. The Netherlands Society for Materials Science 675-680. 